\title{
man \\ Distributed Electric Vehicle Charging Scheduling with Transactive Energy Management
}

\author{
Zhouquan $\mathrm{Wu}^{1, *}$ and Bo Chen ${ }^{1,2}$ \\ 1 Department of Electrical and Computer Engineering, Michigan Technological University, \\ 1400 Townsend Drive, Houghton, MI 49931, USA; bochen@mtu.edu \\ 2 Department of Mechanical Engineering-Engineering Mechanics, Michigan Technological University, \\ 1400 Townsend Drive, Houghton, MI 49931, USA \\ * Correspondence: wzhouqua@mtu.edu
}

check for

updates

Citation: $\mathrm{Wu}, \mathrm{Z}$; Chen, $\mathrm{B}$.

Distributed Electric Vehicle Charging Scheduling with Transactive Energy Management. Energies 2022, 15, 163. https://doi.org/10.3390/en15010163

Academic Editor: Pierluigi Siano

Received: 30 November 2021

Accepted: 23 December 2021

Published: 27 December 2021

Publisher's Note: MDPI stays neutral with regard to jurisdictional claims in published maps and institutional affiliations.

Copyright: (C) 2021 by the authors. Licensee MDPI, Basel, Switzerland. This article is an open access article distributed under the terms and conditions of the Creative Commons Attribution (CC BY) license (https:// creativecommons.org/licenses/by/ $4.0 /)$.
Abstract: A distributed electric vehicle (EV) charging scheduling strategy with transactive energy (TE) management is presented in this paper to deal with technical issues in distribution network operation and discuss the economic benefits of EV charging. At an individual EV level, EV owners propose bids to actively participate in the distribution system operation. At the node level, an electric vehicle aggregator (EVA) optimally allocates the available charging power to meet EV charging requirements and cost benefits. At the distribution network level, a distribution system operator (DSO) integrates an electricity price market clearing mechanism with the optimal power flow (OPF) technique to ensure the reliability of the distribution network. Moreover, a distributed algorithm is discussed for solving the EV charging problem with transactive energy management (TEM). The clearing electricity price is achieved through a negotiation process between the DSO and EVAs using the alternating direction method of multipliers (ADMM). The presented EV charging scheduling with TEM is tested on a modified IEEE 33-bus distribution network scenario with 230 EV charging loads. The simulation results demonstrate the effectiveness of the TE-based EV charging scheduling system.

Keywords: transactive energy; distribution networks; electric vehicle; alternating direction method of multipliers

\section{Introduction}

In recent years, the market share of electric vehicles has rapidly increased. It is estimated that global electric vehicle sales will reach a total of 22 million by 2025 [1]. The charging needs of large-scale electric vehicles could cause significant impacts on the power grid, including overloading, frequency deviation, and voltage violations [2]. To mitigate these impacts, smart EV charging scheduling and control are extensively explored for a microgrid [3-5]. To expand large-scale EV charging in distribution networks, Cao et al. [6] formulated a large-scale EV charging control solution without violating node and substation power limitations. Wang et al. [7] have developed an EV charging control scheme for grid frequency regulation. Cao et al. [8] propose a vehicle-grid integration control strategy for distribution network voltage regulation. All these studies illustrate that constructive EV charging scheduling and control methods have the potential to reduce or eliminate the negative impacts of EV charging on distribution networks. Nevertheless, few existing works have studied both the economic benefits to EV customers of their participation in the grid operation for distribution network stability.

To offer economic benefits, the application of transactive energy in EV charging scheduling has been studied. Transactive energy is a set of mechanisms to balance power generation and consumption through economic values as defined by the GridWise Architecture Council [9]. TE is a multi-agent system that aligns individual behaviors with the interests of the entire system [10]. Lezama et al. [11] have developed a local market to 
coordinate the distribution of energy resources and wholesale markets. Vayá et al. [12] introduced an optimal bidding strategy for EV owners to participate in day-ahead electricity markets. Lakshmi et al. [13] have designed an energy management strategy for the optimal utilization of EV bid fulfillment. The aggregator is responsible for proposing energy bids, controlling the rate of charging/discharging of EVs, and minimizing the operation cost of the microgrid. Gao et al. [14] propose a price-based iterative double auction market mechanism between EV charge stations and EV drivers. The social welfare of both charger owners and EV drivers is maximized in this way. However, these works focus on price mechanisms and lack analysis of distribution power grid operations. Masood Et al. [15] present a transactive energy framework for aggregated EV charging in the local market to reduce the system peak load. Besides the peak load, the optimal operation needs to be considered to ensure that the DSO benefits. Li et al. [16] designed a distributed transactive energy trading framework to integrate photovoltaics. Li et al. [17] developed a hierarchical scheduling method for heating, ventilation, and air-conditioning (HVAC) systems of office buildings as well as for a few electric vehicles. However, large-scale EV charging may cause significant impacts on the distribution network compared with conventional building loads. These works $[16,17]$ have addressed distribution power network operation with the OPF technique, but lack price mechanism analysis. Hao et al. [18] developed transactive controls with a double auction market for commercial buildings. However, it is limited on the microgrid level. Wu et al. [19] explored the TE-based approach for large-scale EV charging, but the market and coordination mechanism are not included. The research towards applying truly transactive energy management, including the economic benefits of market or price mechanisms and the optimal operation of the distribution network, to large-scale EV charging scheduling, is still to be widely explored.

Traditional distribution networks are managed in a centralized manner to ensure the stability of the distribution network through optimal power flow techniques. However, the centralized control of large-scale EV charging in a distribution network is challenging due to the complexity of communication and privacy concerns. Wang et al. [20] have developed a fully distributed large-scale EV charging control strategy based on a consensus algorithm. The total charging power losses are minimized and discharged power from EVs to the grid is maximized. Zhou et al. [21] combined Nash equilibrium and Lyapunov optimization and developed an incentive-based distributed scheduling of EV charging. Yan et al. [22] have designed a model-free deep-reinforcement learning-based approach for an optimal charging control strategy. Khaki et al. [23] have designed a hierarchical distributed framework for EV charging, based on ADMM. The effectiveness of ADMM in transferring a centralized scheduling framework to a decentralized or distributed framework for the distribution network with community energy systems is presented in [24]. The algorithms in [20-23] show the effectiveness of distributed EV charging scheduling and control, but the operation stability of the distribution network is not considered. OPF is widely adapted in power system operations to deal with practical issues. Farivar et al. [25] propose a branch flow model for the analysis of distribution networks. This model offers a new approach to solving OPF in a distributed manner. By combing the OPF techniques with market mechanisms, an economical and reliable distribution system will enable the realization of modern EV charging control with transactive energy management.

In this paper, a distributed EV charging schedule with transactive energy management (TEM) strategy is presented to handle large-scale EV charging in distribution networks. A distributed control system is developed to find a balance between EV charging costs and distribution system operation stability. The contributions of this paper include:

- An innovative EV bidding strategy, designed to encourage individual EV customers to actively participate in the distribution network operation. Only charging demand and price information are shared with EVA and DSO in the bidding process. In this way, the communication complexity is reduced and the system's security is improved.

- A distributed multi-agent coordination algorithm was developed to integrate EV charging optimization with the distribution network OPF technique based on the 
alternating direction method of multipliers (ADMM). In this algorithm, EV charging scheduling and clearing electricity prices are determined through a negotiation process among DSOs and EVAs. EVAs ensure that EV charging requirements and charging cost economics are met, and DSOs guarantee the distribution network operation stability with OPF. The negotiation process finds a balance among EV charging requirements, EV charging economic benefits, and distribution system operation reliability.

- By applying transactive energy management, an EV charging price clearing mechanism is introduced. This mechanism engages EV bidding conditions, distribution system operation, and the electricity market, and then clearing of the electricity price in the negotiation process. The price signal is the only external signal for making the final EV charging decision.

The rest of the paper is organized as follows: Section 2 provides an overview of EV charging scheduling management. Section 3 introduces the EV charging model and distribution network branch flow model. Section 4 formulates a 3-stage EV charging scheduling problem with TEM. Section 5 shows the simulation results of a use case study. Section 6 concludes this paper.

\section{Overview of EV Charging Scheduling Management}

Consider a radial distribution network with $J$ buses, a DSO is designed at the slack bus to ensure grid stability and address the economic benefits. In addition, several EVAs are located at each node except the slack bus. Each EVA responds to a group of EVs based on their physical locations. As shown in Figure 1, the system includes multi-criterion optimizations among the DSO, EVAs, and individual EVs.

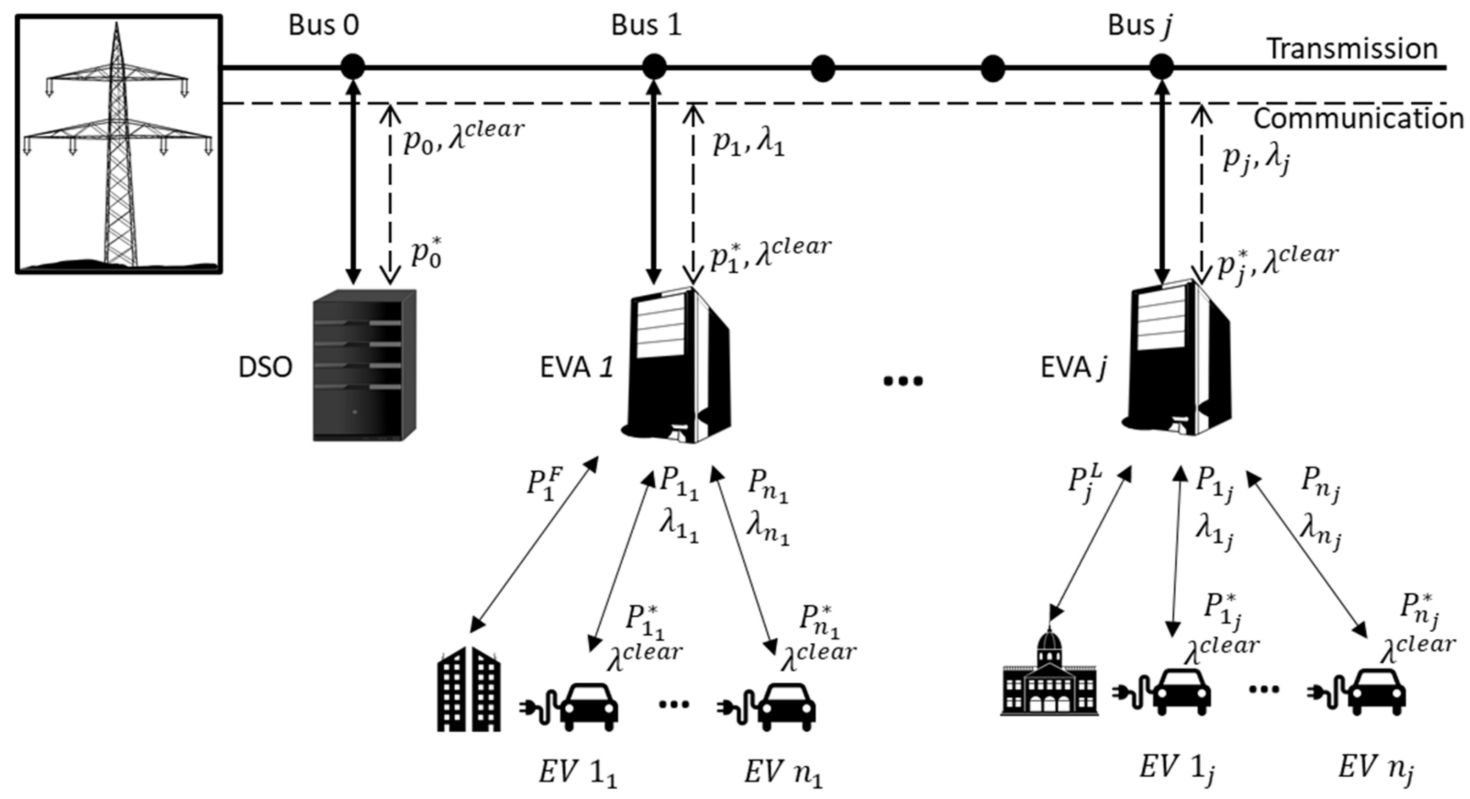

Figure 1. The system diagram of TE-based EV charging.

To coordinate the operation of the integrated EVs, EVAs, and DSO, a TE-based EV charging mechanism is designed to achieve a reliable and cost-efficient electricity system. In contrast to the traditional demand response control, EV customers can choose to buy and prioritize the charging cost. As shown in Figure 2, EV charging scheduling management comprises three stages. In Stage 1, an optimal EV bidding strategy of individual EV and node-level aggregation is designed. Individual EV owners will propose their optimal bidding strategy based on day-ahead electricity price forecasts and charging requirements. Then, a node-level EVA will collect all bidding information within the node and form a bidding price for the node. With this strategy, EVs can directly participate in the distribution- 
level market clearing mechanism in Stage 2. In Stage 2, the DSO will clear the electricity bidding and find the clearing electricity price, and EVA will adjust its feasible power demand. Meanwhile, the OPF technique will be addressed in this stage to ensure the smooth operation of the grid. In Stage 3, the node-level EVA will optimally determine the charging rate for individual EVs within the node by considering EV owners' bidding demands in Stage 1, the clearing electricity prices, and the available power in the node.

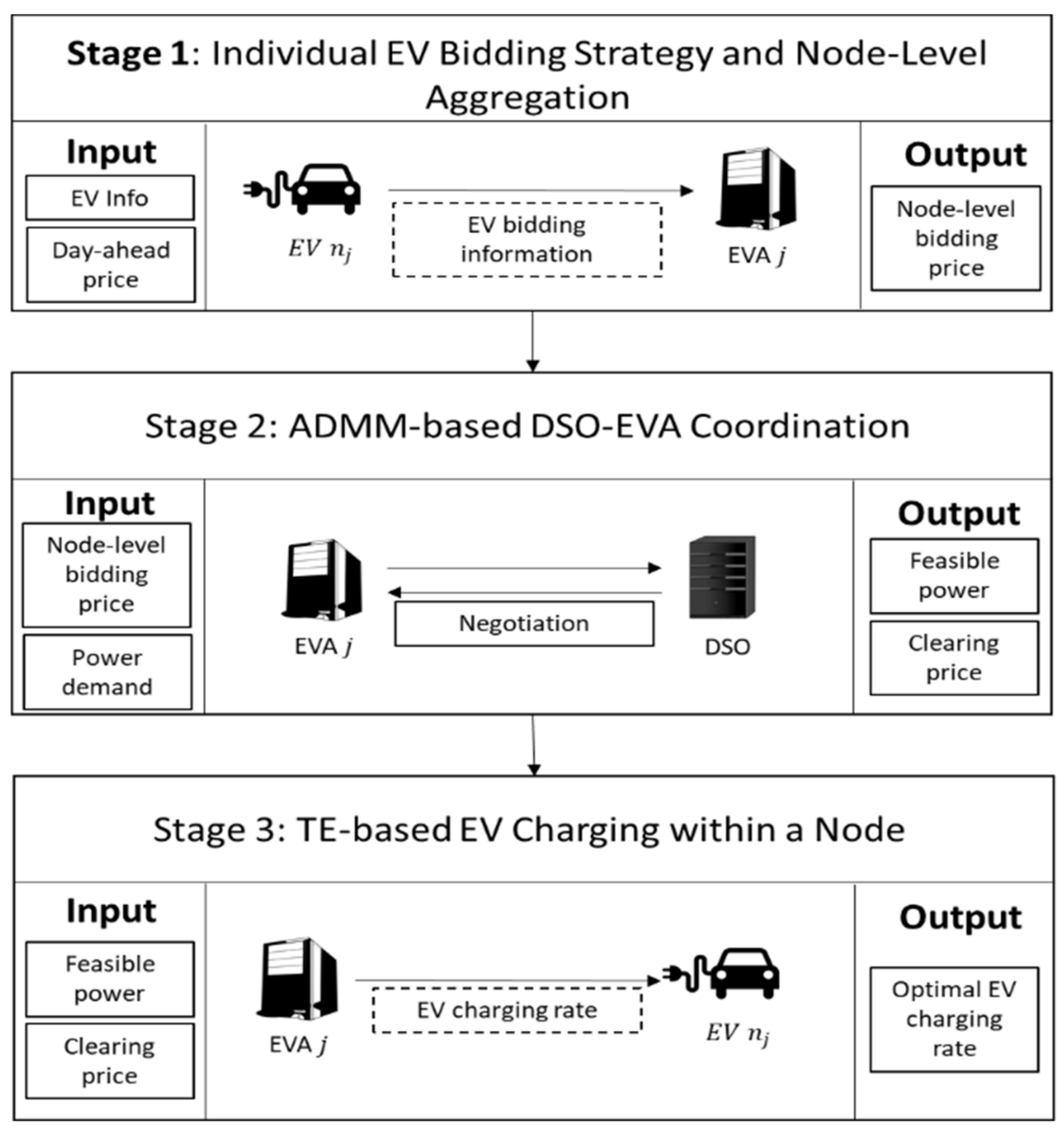

Figure 2. Three-stage EV charging scheduling management.

\section{EV Charging and Distribution Network Model}

\subsection{EV Charing Model}

An individual EV seeks to optimize its charging power scheduling for a time period of $T$ as defined by a vector of $\boldsymbol{P}_{n_{j}}=\left[P_{n_{j}}(1), \ldots, P_{n_{j}}(t), P_{n_{j}}(t+1), \ldots, P_{n_{j}}(T)\right]$, where $n_{j} \in\left\{1_{j}, \ldots, N_{j}\right\}$ represents the location of $n^{\text {th }}$ EV supplied by EVA $j$. The EV charging process is modeled as a discrete-time linear system as:

$$
\operatorname{SoC}_{n_{j}}(t+1)=\operatorname{SoC}_{n_{j}}(t)+\frac{\eta_{n_{j}} P_{n_{j}}(t) \Delta t}{E_{n_{j}}^{C}}
$$

where $P_{n_{j}}(t)$ is the charging power at time step $t, E_{n_{j}}^{C}$ indicates the battery capacity, $\eta_{n_{j}}$ is the charging efficiency, and $\Delta t$ represents the time step duration. The energy requirement, maximal and minimal charger power rating of individual EVs are defined as a series of 
constraints. It is assumed that the charging duration and energy requirement are known as soon as EVs connect to the charging stations. The constraints of individual EV charging schedules are:

$$
\begin{gathered}
\begin{cases}P_{n_{j}}^{\min } \leq P_{n_{j}}(t) \leq P_{n_{j}}^{\max } & \text { if active } \\
P_{n_{j}}(t)=0 & \text { if inactive }\end{cases} \\
\operatorname{SoC}_{n_{j}}\left(t+d_{n_{j}}\right)=S o C_{n_{j}}^{T}
\end{gathered}
$$

Vehicle-to-grid power flow is not considered in this paper. Therefore, the values $P_{n_{j}}^{\max }$ and $P_{n_{j}}^{\min }$ indicate the maximal and minimal EV charging rate for the time horizon. The parameter $d_{n_{j}}$ denotes the number of time steps remaining until departure and $S o C_{n_{j}}^{T}$ is the target $\mathrm{SoC}$ by the next departure.

\subsection{Distribution Network Model}

Given a radial distribution network represented by graph $G=(J, E), J=\{0, \ldots, J\}$ represents the set of feeder nodes and $E$ denotes the set of lines between the buses in the network, e.g., $(i, j) \in E$ indicates the line that from bus $i$ to bus $j$. Let bus 0 represent the substation bus that connects to the utility as an external power source. The branch flow model in a radial distribution network is shown in Figure 3.

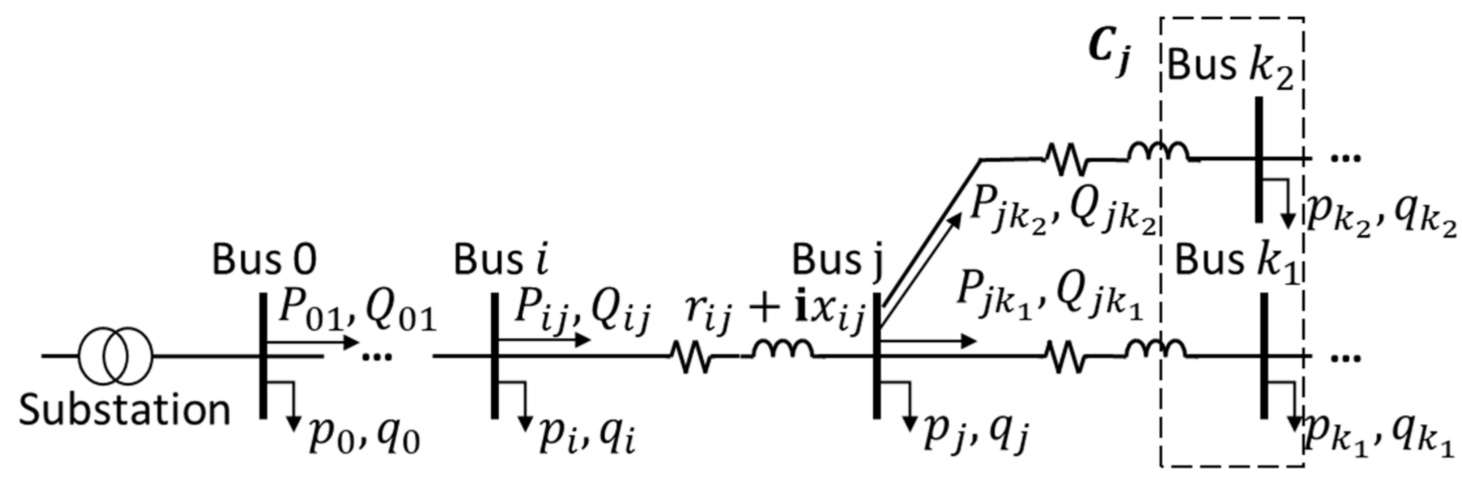

Figure 3. The branch flow model in a radial distribution network.

For each node $j \in J$, let $p_{j}$ and $q_{j}$ be the active and reactive injection power, and $V_{j}$ denote the complex voltage on this bus. For each link $(i, j) \in E, r_{i j}$ and $x_{i j}$ are the resistance and reactance of this line; $I_{i j}$ represents the complex current; $P_{i j}$ and $Q_{i j}$ denote the sending-end complex power from bus $i$ to $j$. Except for the substation bus (indexed as 0 ), each node $j$ has a unique parent node $i$ and a set of child nodes $C_{j}$. Let $l_{i j}=\left|I_{i j}\right|^{2}$ denote the squared line current magnitude of the line $(i, j)$ and $v_{j}=\left|V_{j}\right|^{2}$ be the squared voltage magnitude of node $j$. The power balance and flow equations of the distribution network can be formulated as [26]:

$$
\begin{gathered}
p_{j}(t)=P_{i j}(t)-r_{i j} l_{i j}(t)-\sum_{k \in C_{j}} P_{j k}(t), \forall j \in J \\
q_{j}(t)=Q_{i j}(t)-x_{i j} l_{i j}(t)-\sum_{k \in C_{j}} Q_{j k}(t), \forall j \in J \\
v_{j}(t)=v_{i}(t)-2\left(r_{i j} P_{i j}(t)+x_{i j} Q_{i j}(t)\right)+\left(r_{i j}^{2}-x_{i j}^{2}\right) l_{i j}(t), \forall(i, j) \in E \\
l_{i j}(t)=\frac{P_{i j}(t)^{2}+Q_{i j}(t)^{2}}{v_{i}(t)}, \quad \forall(i, j) \in E
\end{gathered}
$$


However, the quadratic equalities in (5) will cause non-convex optimization, which is difficult to solve and does not guarantee convergence. Thus, a second-order cone relaxation [25] is applied as the inequality constraints:

$$
l_{i j}(t) \geq \frac{P_{i j}(t)^{2}+Q_{i j}(t)^{2}}{v_{i}(t)}, \quad \forall(i, j) \in E
$$

The OPF problem will be transformed to a convex one for radial networks. Meanwhile, to satisfy this relaxation, the voltage of buses should be very close to the nominal value and the power input to the bus should be under a certain limit. The additional constraints to ensure the prescribed region are:

$$
\begin{aligned}
& v_{j}^{\min } \leq v_{j}(t) \leq v_{j}^{\max }, \quad \forall j \in J \\
& 0 \leq l_{i j}(t) \leq l_{i j}^{\max }, \quad \forall(i, j) \in E
\end{aligned}
$$

(7) prevents voltage violation, and (8) limits the current of each line $i j$ respectively. The values $v_{j}^{\min }$ and $v_{j}^{\max }$ are the minimum and maximum of voltage magnitude, and $l_{i j}^{\max }$ is considered as the maximum of the current magnitude.

\section{Problem Formulation of Distributed EV Charging Scheduling with Transactive Energy Management}

\subsection{Individual EV Bidding Strategy and Node-Level Aggregation}

The framework for individual EV bidding strategy and node-level aggregation is shown in Figure 4. Firstly, according to the charging request, demand, and duration, individual EVs will find the optimal bidding strategy and forward it to the EVA. Then, to coordinate with others, the EVA needs to form the final bidding price and netload demand.

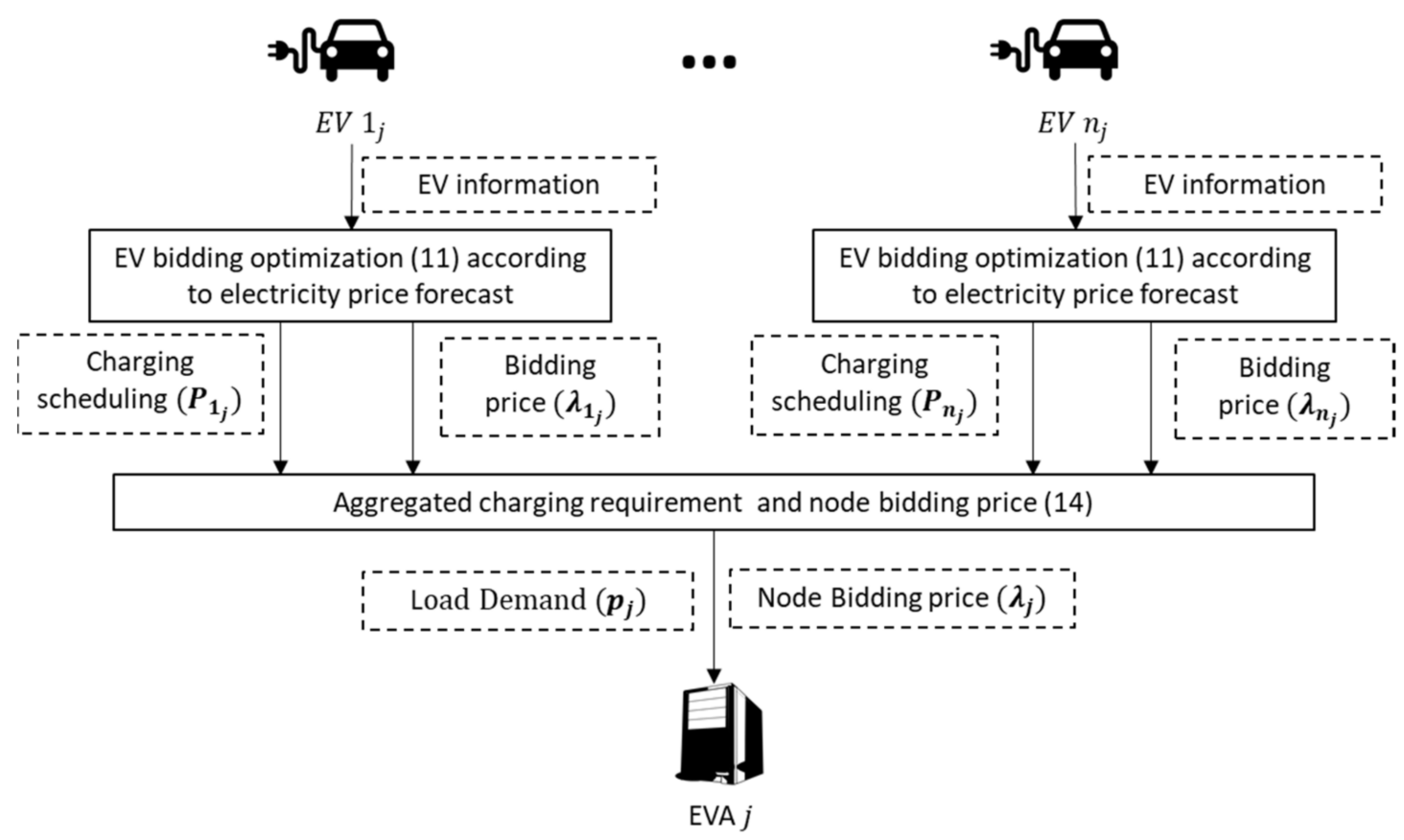

Figure 4. EV bidding and node-level aggregation.

Individual EV owners need to propose a bidding price to compete with others. To be fair, a blind auction mechanism is considered in the bidding process. The bidding price is defined by a vector $\lambda_{n_{j}}=\left[\lambda_{n_{j}}(1), \ldots, \lambda_{n_{j}}(t), \lambda_{n_{j}}(t+1), \ldots, \lambda_{n_{j}}(T)\right]$. At each time step, the bidding price reflects both the current charging power demand and the remaining charging 
flexibility. For example, an EV owner whose vehicle has a longer charging duration and a lower energy requirement is not inclined to offer a high price to obtain more charging power. Thus, the preferred charging electricity price $\lambda_{n_{j}}(t)$ is formulated as:

$$
\begin{gathered}
\lambda_{n_{j}}(t)=\lambda^{D A}(t)+\frac{\lambda^{\operatorname{Cap}}(t)}{P_{n_{j}}^{\max }-P_{n_{j}}^{\min }} \mu_{n_{j}}(t)\left(P_{n_{j}}(t)-P_{n_{j}}^{\min }\right) \\
\mu_{n_{j}}(t)=\min \left[\frac{\left(S o C_{n_{j}}^{T}(t)-S o C_{n_{j}}(t)\right) E_{n_{j}}^{C}}{\eta_{n_{j}} P_{n_{j}}^{\max } d_{n_{j}} \Delta t}, 1\right]
\end{gathered}
$$

At time step $t$, the day-ahead electricity price $\lambda^{D A}$ and the feasible increase range of the preferred charging electricity price $\lambda^{\mathrm{Ca} a}$ are assumed to be forecasted by the utility. The charging urgency $\mu_{n_{j}}$ is defined in (10) to reflect the flexibility of the remaining charging duration $d_{n_{j}} \times \Delta t$.

Once the pricing principle was defined, individual EV owners will propose their bidding schedule without negotiation with others. Each EV customer pursues a target charging cost. The optimization formulation is given below:

$$
\begin{array}{ll}
\min & \boldsymbol{\lambda}_{n_{j}} \cdot \mathbf{P}_{n_{j}}^{T} \cdot \Delta t \\
\text { s.t. } & (1)-(3) \\
& \mathbf{P}_{\mathbf{n}_{\mathbf{j}}}, \forall n_{j} \in N_{j} \\
C_{n_{j}}= & \lambda_{n_{j}}^{*} \cdot \mathbf{P}_{n_{j}}^{* T} \cdot \Delta t
\end{array}
$$

where $C_{n_{j}}$ indicates the target optimal cost during the bidding process, while $\mathbf{P}_{n_{j}}^{*}$ and $\lambda_{n_{j}}^{*}$ are the optimal power demand and bidding price solved by problem (11). These only depend on the day-ahead electricity price forecast and individual EV charging requirements. The results can be solved with quadratic programming and selected as the target of initial charging cost in stage 3 .

The EVA serves as the bridge between individual EVs and the DSO. In stage 1, the EVA aggregates power demand and finds bidding prices for all the EVs in the node. The aggregated bidding price will inform the DSO for market clearing via the communication shown in Figure 1. For individual EVA $j \in\{1, \ldots, J\}$, where $N$ denotes the number of EVAs in the distribution network, the aggregated power demand can be formulated by:

$$
\mathbf{p}_{\mathbf{j}}=\mathbf{P}_{\mathbf{j}}^{\mathbf{F}}+\sum_{n \in \mathbf{N}_{\mathbf{j}}} \mathbf{P}_{\mathbf{n}_{\mathbf{j}}}
$$

Let $\mathbf{p}_{\mathbf{j}}=\left[p_{j}(1), \ldots, p_{j}(T)\right]$ be the aggregated active power demand by EVA for bus $j$, and $\mathbf{P}_{\mathbf{j}}^{\mathbf{F}}$ is the vector of aggregated uncontrollable load demand. The aggregated bidding price for EVA $j$ at time step $t$ is:

$$
\lambda_{j}(t)=\sum_{n \in \mathbf{N}_{\mathbf{j}}}\left(\lambda_{n_{j}}(t) \frac{P_{n_{j}}(t)}{\sum_{n \in \mathbf{N}_{\mathbf{j}}} P_{n_{j}}(t)}\right)
$$

where the bidding price is denoted by $\lambda_{j}$. It is designed as the expected value of individual EV owners' bidding prices in proportion to the amount of power demand.

\subsection{ADMM-Based DSO-EVA Coordination}

Figure 5 shows the negotiation process between the DSO and EVAs to determine the optimal clearing prices $\lambda^{*}$ for the distribution network and optimal feasible netload $\boldsymbol{p}_{j}^{*}$ for each EVA. 


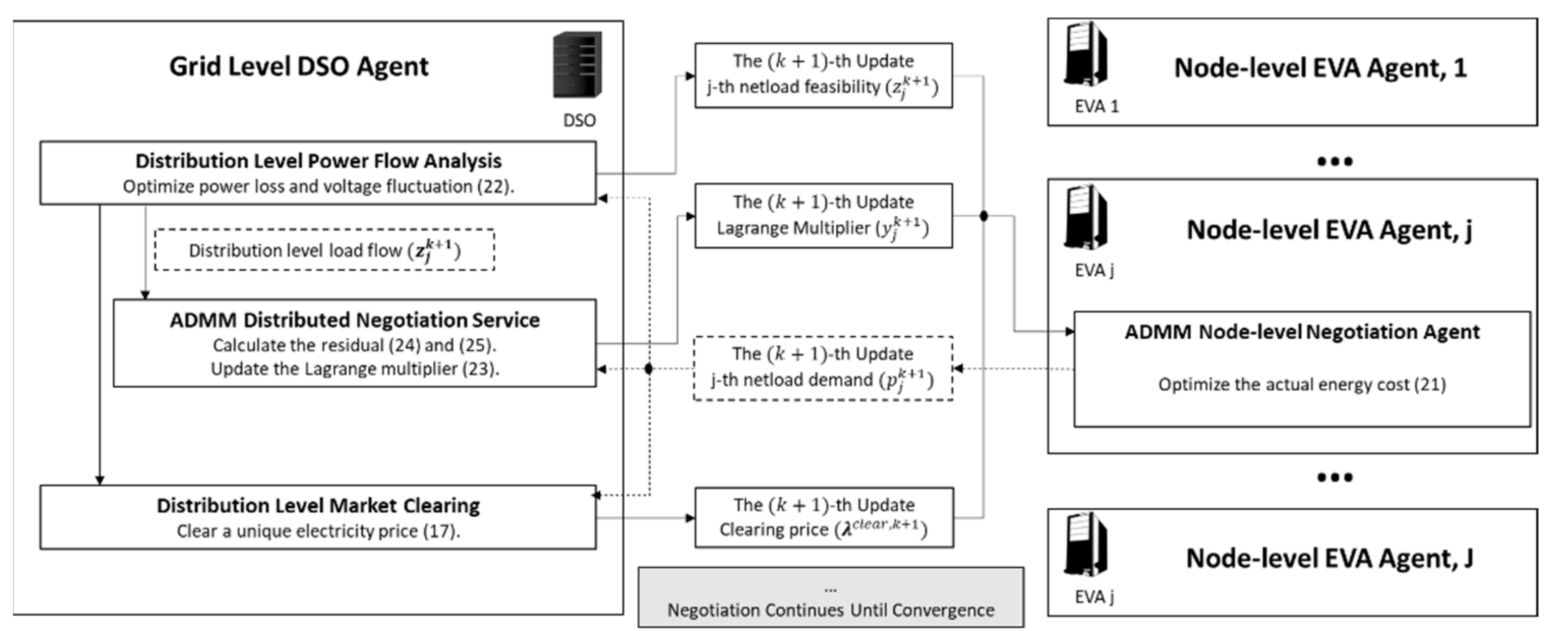

Figure 5. The framework of ADMM-based DSO-EVA Coordination.

The EVA adjusts its power demand according to the clearing price and re-negotiates with the DSO. EVAs aim to reduce the charging cost in terms of group EVs, so the objective function can be formatted as:

$$
f_{j}\left(\mathbf{p}_{\mathbf{j}}\right)=\lambda^{\text {clear }} \cdot \mathbf{p}_{j}^{T} \cdot \Delta t
$$

where $\lambda^{\text {clear }}$ indicates the vector of clearing electricity price proposed by the DSO during the negotiation.

The DSO is located at the substation bus 0 as shown in Figure 1. It purchases electricity from the utility and ensures the reliability of the distribution network using OPF techniques. The objective function of DSO is designed to reduce the cost due to energy loss as shown below.

$$
g\left(\mathbf{p}_{\mathbf{j}}\right)=\lambda^{D A} \cdot \sum_{i j \in E}\left(\mathbf{l}_{\mathbf{i j}}^{T} \cdot \mathbf{r}_{\mathbf{i j}}\right) \cdot \Delta t
$$

where $\mathbf{l}_{\mathbf{i j}}=\left\{l_{i j}(1), \ldots, l_{i j}(t), l_{i j}(t+1), \ldots, l_{i j}(T)\right\}$ indicates a vector of the square of line current magnitude for a time period of $T \times \Delta t$. The DSO also provides the functions of a competitive market for this distribution network. It collects bidding price information from EVAs and clears a unique electricity price to coordinate the power schedules for EVAs. During negotiation, the clearing electricity price at time step $t$ is given by:

$$
\lambda^{\text {clear }}(t)=\frac{\sum_{j \neq 0: j \in \mathbf{N}}\left(\lambda_{j}(t) p_{j}(t)\right)}{p_{0}(t)}
$$

where $p_{0}$ and $\boldsymbol{q}_{0}$ represent the vector of active and reactive power purchase from the utility

In this radial distribution system with a DSO and multi-EVAs, the goal of the DSO is to minimize energy loss, while EVAs prefer to reduce the actual charging cost. The optimal DSO-EVA coordination problem can be formulated as:

$$
\begin{array}{ll}
\min & \mathrm{g}\left(\mathbf{p}_{\mathbf{j}}\right)+\sum_{j=1}^{J} f_{j}\left(\mathbf{p}_{\mathbf{j}}\right) \\
\text { over } & \mathbf{p}_{\mathbf{j}} \forall j \in J \\
\text { s.t. } & (4),(6),(7),(8) \\
& \mathbf{p}_{j}^{\min } \leq \mathbf{p}_{j} \leq \mathbf{p}_{j}^{\max }
\end{array}
$$

where $\mathbf{p}_{j}^{\min }$ and $\mathbf{p}_{j}^{\text {max }}$ represent the minimum and maximum load for each EVA. 
Although the optimization problems (18) can be solved by a centralized method, it is not computationally efficient and will cause privacy issues since all the information needs to be transmitted and processed by the DSO. The decision variables for the DSO are strongly coupled with each EVA's decision variable. By introducing an auxiliary variable $\mathbf{z}_{j}$, the ADMM method [27] can be used to solve the problem in a distributed manner. The problem (18) can be redefined as:

$$
\begin{array}{ll}
\min & \mathbf{g}\left(\mathbf{z}_{\mathbf{j}}\right)+\sum_{j=1}^{J} f_{j}\left(\mathbf{p}_{\mathbf{j}}\right) \\
\text { s.t. } & \mathbf{p}_{j}=\mathbf{z}_{j} \forall j \in J \\
& (4),(6),(7),(8) \text { for DSO } \\
& \mathbf{p}_{j}^{\text {min }} \leq \mathbf{p}_{j} \leq \mathbf{p}_{j}^{\max } \text { for EVA }
\end{array}
$$

where the auxiliary variable $\mathbf{z}_{j}=\mathbf{p}_{j}$ proposed by the DSO is to link the injection power $\mathbf{p}_{j}$ of each node. The augmented Lagrange function is defined as:

$$
L_{p}\left(\mathbf{p}_{\mathbf{j}}, \mathbf{z}_{\mathbf{j}}, \mathbf{y}\right)=\sum_{j=1}^{J} f_{j}\left(\mathbf{p}_{\mathbf{j}}\right)+\mathrm{g}\left(\mathbf{z}_{\mathbf{j}}\right)+\sum_{j=0}^{J}\left(\mathbf{y}_{j}^{T}\left(\mathbf{p}_{\mathbf{j}}-\mathbf{z}_{\mathbf{j}}\right)+\frac{\rho}{2}\left\|\mathbf{p}_{\mathbf{j}}-\mathbf{z}_{\mathbf{j}}\right\|_{2}^{2}\right)
$$

where $\mathbf{y}_{j}$ is a vector of Lagrange multiplier and $\rho>0$ denotes the penalty parameter. Therefore, the optimization problem (19) can be solved concurrently by each EVA and DSO. At iteration $k$, each EVA solves the problem:

$$
\begin{aligned}
& \mathbf{p}_{j}^{k+1}=\operatorname{argmin}\left(\mathrm{f}_{j}\left(\mathbf{p}_{j}\right)+\mathbf{y}_{j}^{k T} \mathbf{p}_{j}+\frac{\rho}{2}\left\|\mathbf{p}_{j}-\mathbf{z}_{j}^{k}\right\|_{2}^{2}\right) \\
& \text { s.t. } \mathbf{p}_{j}^{\min } \leq \mathbf{p}_{j} \leq \mathbf{p}_{j}^{\max }
\end{aligned}
$$

The DSO solves the problem (22) to address the OPF. Meanwhile, it is also responsible for updating the auxiliary vector $\mathbf{z}_{j}$ through the problem (23) to coordinate with EVAs.

$$
\begin{aligned}
& \mathbf{z}_{j}^{k+1}=\operatorname{argmin}\left(g\left(\mathbf{z}_{j}\right)+\sum_{j=1}^{J}\left(-\mathbf{y}_{j}^{k T} \mathbf{z}_{j}+\frac{\rho}{2}\left\|\mathbf{p}_{j}^{k+1}-\mathbf{z}_{j}\right\|_{2}^{2}\right)\right) \\
& \text { s.t. (4), (6), (7), (8) } \\
& \qquad \mathbf{y}_{j}^{k+1}=\mathbf{y}_{j}^{k}+\rho\left(\mathbf{p}_{j}^{k+1}-\mathbf{z}_{j}^{k+1}\right)
\end{aligned}
$$

The ADMM iteration satisfies the residual, objective, and dual variable convergence. Given by the residuals of primal feasibility $r$ and dual feasibility s, the stopping criteria is defined as:

$$
\begin{aligned}
& \left\|r^{k}\right\|_{2}^{2}=\sum_{j=1}^{J}\left\|\mathbf{p}_{j}^{k}-\mathbf{z}_{j}^{k}\right\|_{2}^{2} \leq \varepsilon^{p r i} \\
& \left\|s^{k}\right\|_{2}^{2}=\rho\left\|\mathbf{z}_{j}^{k}-\mathbf{z}_{j}^{k-1}\right\|_{2}^{2} \leq \varepsilon^{d u a l}
\end{aligned}
$$

where $\varepsilon^{p r i}$ and $\varepsilon^{\text {dual }}$ are feasibility tolerances for the primal and dual residuals.

\subsection{TE-based EV Charging within a Node}

After the DSO-EVA negotiation process, each EVA will receive the optimal feasible power $\boldsymbol{p}_{j}^{*}$ and clearing prices $\lambda^{*}$ from stage 2 . Figure 6 shows the process of optimal EV charging management within a node.

Individual EVs want to track the target charging $\operatorname{cost} C_{n_{j}}$ in stage 1 because it is the optimal solution without considering any distribution network congestion. Therefore, the EVA needs to generate a sequence of actual EV charging rates for each EV under the node 
to meet this objective. In this paper, the objective function of EV charging for a group of EVs under the EVA $j$ is defined as:

$$
\begin{array}{ll}
\min & \sum_{n=1}^{N_{j}}\left(\lambda^{*} \cdot \mathbf{P}_{\mathbf{n}_{\mathbf{j}}}{ }^{T} \cdot \Delta t-C_{n_{j}}\right)^{2} \\
\text { over } & \mathbf{P}_{n_{j}}, \forall n_{j} \in N_{j} \\
\text { s.t. } & (1)-(3)
\end{array}
$$

The optimal solutions of individual EV charging rate schedule $\boldsymbol{p}_{n_{j}}^{*}$ can be solved by the quadratic programming method and the EVA will send this information to all EV charging stations in the region.
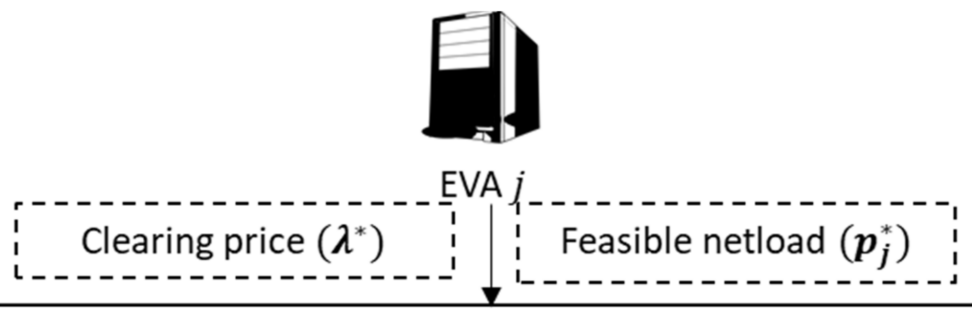

TE-based EV charging optimization (26) according to bidding cost from Stage 1

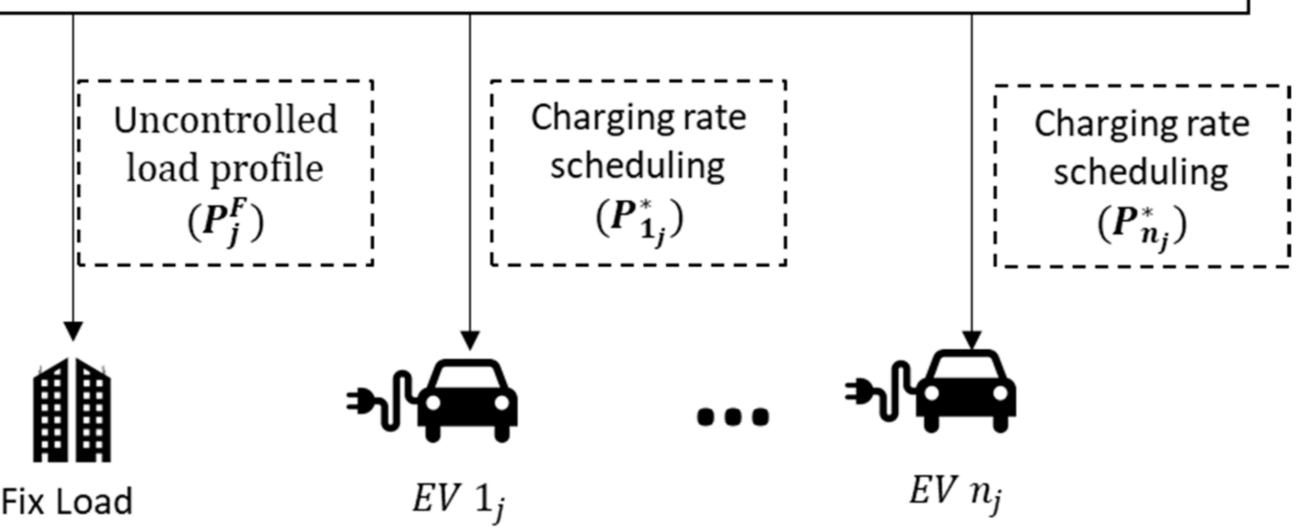

Figure 6. EV Charging optimization within a Node.

\section{Use Case Study}

To validate the presented TE-based EV charging scheduling management algorithm, a modified IEEE 33-bus system [27] is selected to simulate a medium-voltage distribution network. The distribution nodes are classified into residential nodes and commercial nodes based on their load patterns. Different building load profiles are obtained from [28] and modeled into each node. The nominal voltage of the 33-bus distribution system is $12.88 \mathrm{kV}$ and the topology is shown in Figure 7. In addition to building loads, a total of $230 \mathrm{EV}$ charging loads are modeled in 14 nodes. The battery capacity $E_{n_{j}}^{C}$ is $40 \mathrm{kWh}$, the minimal charge rate $P_{n_{j}}^{\min }$ is $1.44 \mathrm{~kW}$, and the maximum charge rate $P_{n_{j}}^{\max }$ is $6.6 \mathrm{~kW}$. The $\mathrm{EV}$ arrival/departure time and initial/target SOC are generated based on [29]. Most EVs charging at commercial areas will continue for two to four hours during the daytime. EVs charging at residential areas will start in the evening or at late night and will end by 6:30 a.m. Without considering the service fee, the day-ahead electricity prices are obtained from [30] as shown in Figure 8 and the feasible increasing range $\lambda^{C a p}$ is set as ${ }^{\star} 2 / \mathrm{kWh}$. The length of the entire horizon is $24 \mathrm{~h}$ (7:00 a.m.-7:00 a.m.) and the duration of each time step is $15 \mathrm{~min}$. 


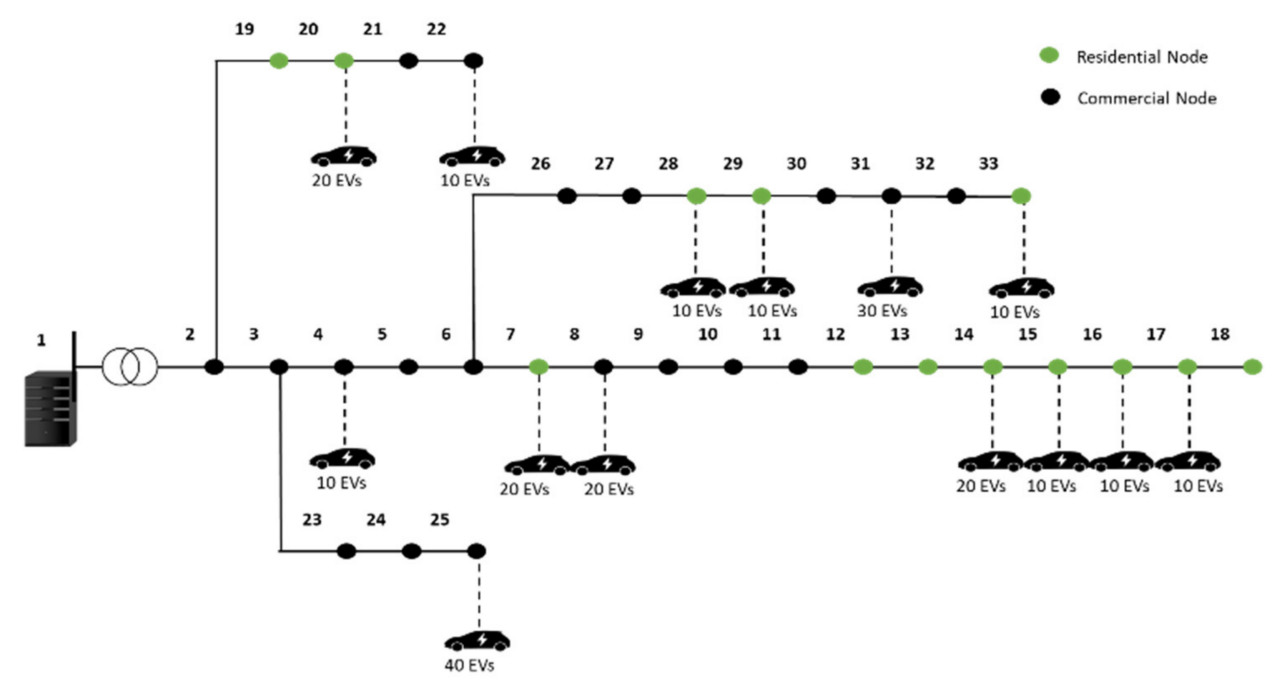

Figure 7. IEEE 33-bus distribution system with EV charging loads.

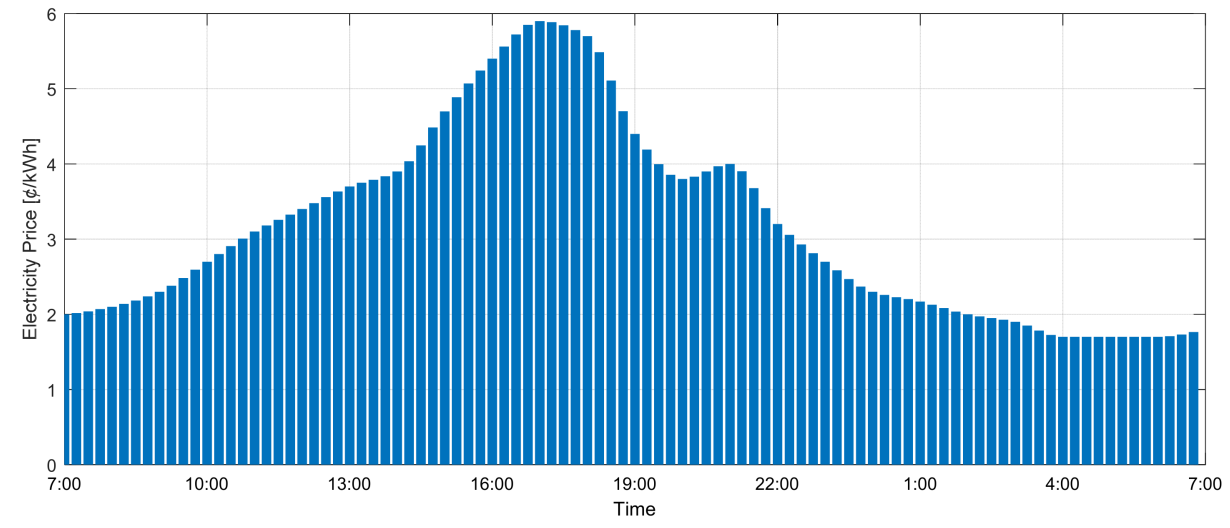

Figure 8. Day-ahead Electricity Price.

From the perspective of the distribution network, Figure 9 shows the load profiles of the 33-bus system with and without TEM. Without TEM, the overall load will exceed the substation capacity in the morning and evening when a large number of EVs are connected. However, the charging loads are shifted smoothly when TEM is introduced so that the load profile is always under the substation capacity. From Figure 9, it is obvious that residential EV charging loads in the peak period (17:00-20:00) are moved to the off-peak period (24:00-6:00). Figure 10 shows the voltage profile of the 33-bus system with TEM. No voltage violation is observed because the coordination of the DSO and EVAs ensures the stability of the distribution network. These results show that TE-based EV charging management can avoid load congestion and improve grid performance.

From the perspective of nodes with EV charging loads, two representative nodes are selected to demonstrate the results. In particular, Node 4 is a commercial node with $10 \mathrm{EV}$ charging loads, and Node 17 represents a residential node with $10 \mathrm{EV}$ charging loads. Figure 11 shows the load profile and EV charging results of commercial Node 4 . With TEM, part of the EV charging load is shifted to avoid exceeding the upper power bound of the node. At the same time, all EV charging requirements are met within their charging durations. Figure 12 shows the load profile and EV charging results of residential Node 17. After applying TEM, almost all EV charging loads are shifted from the peak period to an off-peak period. Residential EV charging has more flexibility to address its charging schedule as well as provide grid service. Meanwhile, all EVs' charging requirements are satisfied. Therefore, TE-based EV charging management is feasible for individual nodes and EVs. 


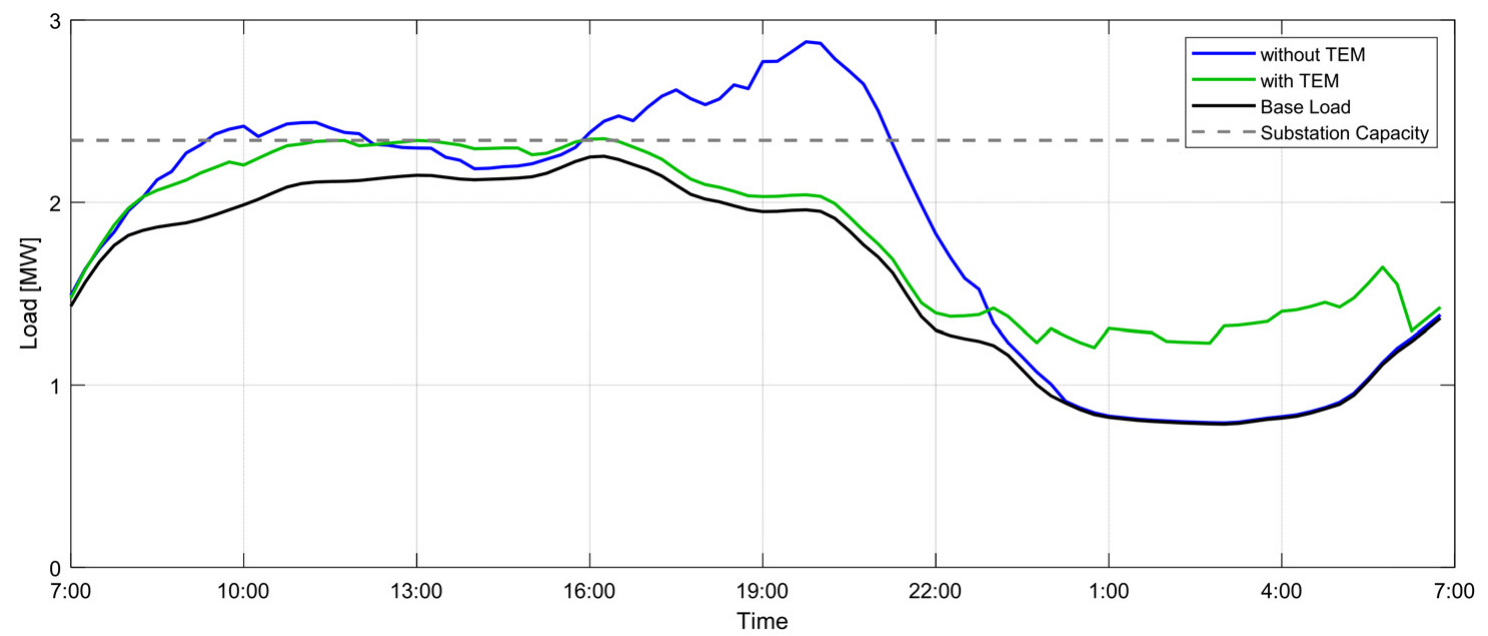

Figure 9. Load profiles of 33-bus system with and without TEM.

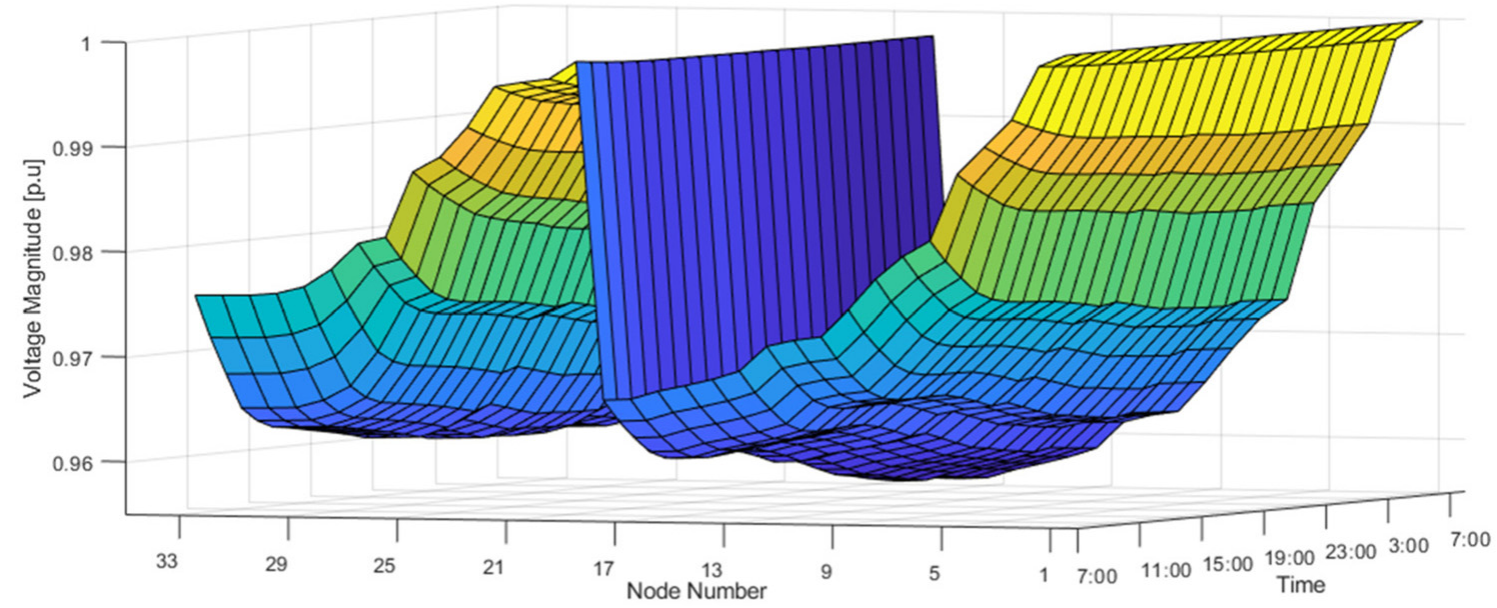

Figure 10. Voltage magnitudes of 33-bus system with TEM.

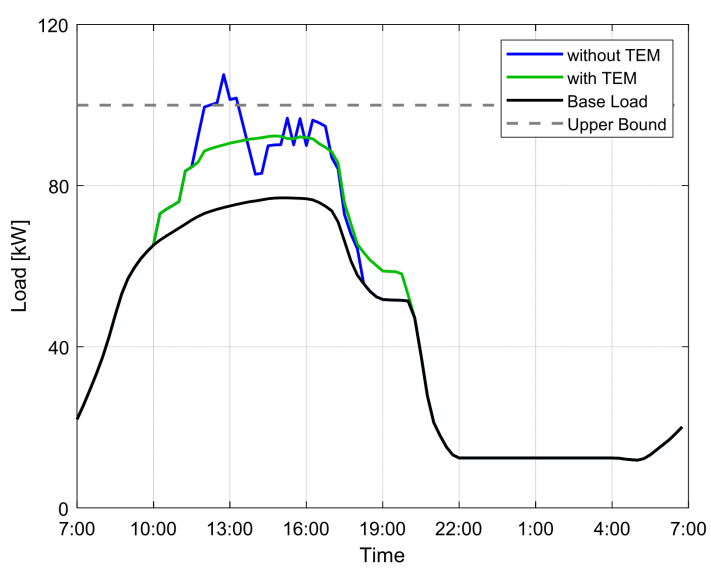

(a)

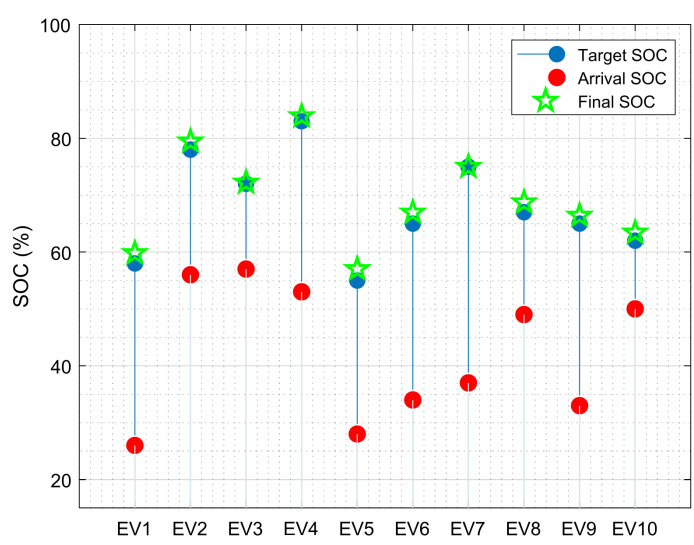

(b)

Figure 11. Load profile and EV charging results of Node 4 (a) Load profile; (b) EV charging results. 


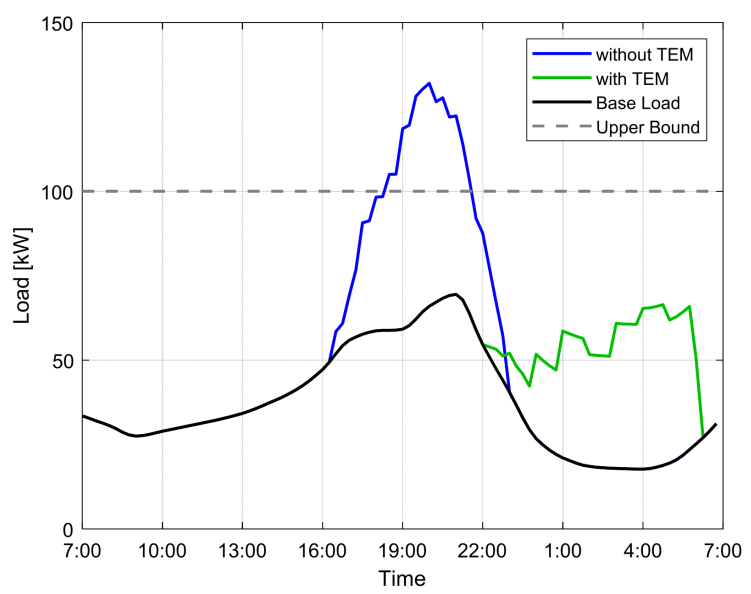

(a)

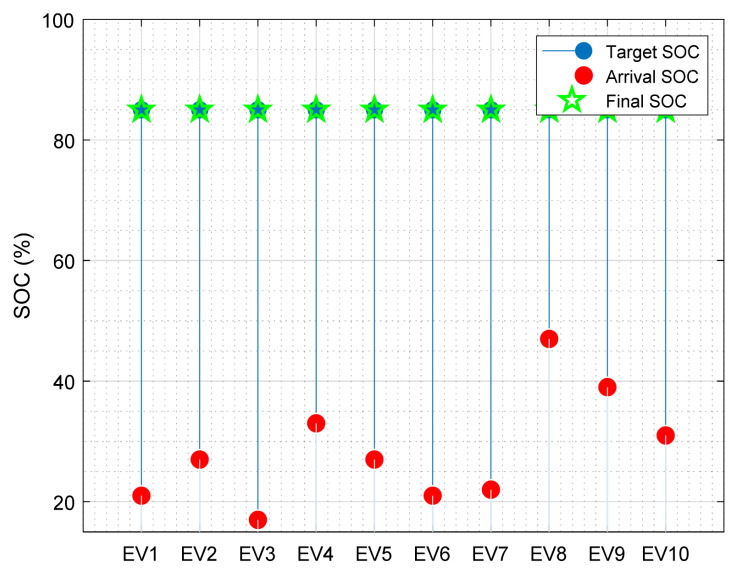

(b)

Figure 12. Load profile and EV charging results of Node 17 (a) Load profile; (b) EV charging results.

Besides ensuring grid stability, TEM also considers customers' charging costs. Figure 13 shows the final EV charging price of EV charging with and without TEM. With TEM, to encourage EV owners to shift their charging demand to support grid operation, such as via voltage regulation and congestion management, the DSO will reduce the EV charging-electricity price in the market clearing mechanism. This incentive mechanism offers better economic benefits for any EVs participating in the TEM program. Table 1 lists the groups of EV charging costs with and without TEM. The results show that TEM can reduce about $39.55 \%$ cost for $230 \mathrm{EVs}$ through a clearing price. Typically, at residential nodes, EVs can save more than $50 \%$ of their charging cost because of overnight charging periods. The charging of residential EVs presents more flexibility to be scheduled according to clearing prices and grid peak/off-peak periods. At commercial nodes, although EVs have less flexibility to respond to clearing prices, TEM can still result in about $10-20 \%$ cost saving for connected EVs. As a result, TE-based EV charging management can also offer economic benefits for distribution networks, nodes, and individual EVs.

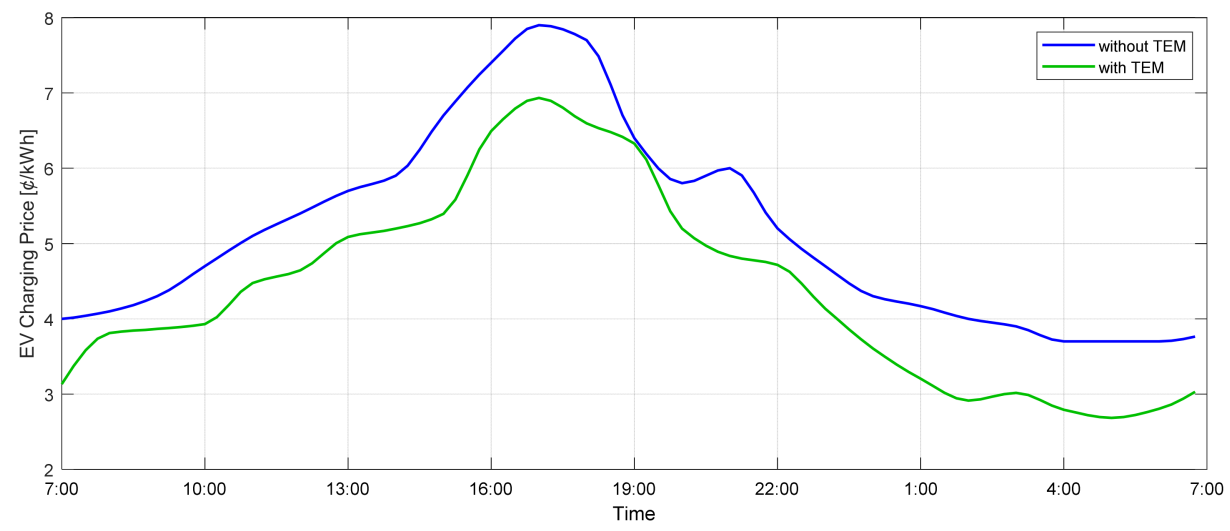

Figure 13. EV charging price with and without TEM.

The presented method is simulated using MATLAB on a laptop with an Intel Core i7 of 2.8 GHz. The optimization problems are formulated using YALMIP [31] with CPLEX as the solver. Figure 14 shows the convergence process for solving the ADMM-based DSO-EVA coordination problem with the parameter values of $\rho=1$ and $\varepsilon^{p r i}=\varepsilon^{d u a l}=0.01$. The norms of primal residual and dual residual converge to 0.01 within 244 iterations. The optimization problem has a large number of variables and constraints due to large-scale 
EVs and EVAs. Additional methods, such as the dynamic step size modification method introduced in [24], will be explored in the future to improve computational efficiency.

Table 1. EV Charging cost of the 33-bus system with and without TEM.

\begin{tabular}{|c|c|c|c|c|c|}
\hline Node Type & Node Number & Number of EV & $\begin{array}{c}\text { Cost with TEM } \\
{\left[{ }^{\mathbb{}}\right]}\end{array}$ & $\begin{array}{c}\text { Cost without TEM } \\
{\left[{ }^{\natural}\right]}\end{array}$ & $\begin{array}{c}\text { Cost Reduction } \\
{[\%]}\end{array}$ \\
\hline \multirow{5}{*}{ Commercial } & 4 & 10 & 685.54 & 778.54 & 11.95 \\
\hline & 8 & 20 & 1732.07 & 1566.27 & 9.57 \\
\hline & 22 & 10 & 445.9 & 568.7 & 21.59 \\
\hline & 25 & 40 & 1990 & 2320.68 & 14.25 \\
\hline & 31 & 30 & 2200.31 & 2432.68 & 9.55 \\
\hline \multirow{9}{*}{ Residential } & 7 & 20 & 1404.41 & 3049.55 & 53.95 \\
\hline & 14 & 20 & 2846.94 & 1366.07 & 52.02 \\
\hline & 15 & 10 & 1551.46 & 7045.9 & 54.59 \\
\hline & 16 & 10 & 629.12 & 1405.74 & 55.25 \\
\hline & 17 & 10 & 736.43 & 1589.63 & 53.67 \\
\hline & 20 & 20 & 1518.65 & 2959.82 & 48.69 \\
\hline & 28 & 10 & 647.3 & 1361.66 & 52.46 \\
\hline & 29 & 10 & 695.28 & 1425.7 & 51.23 \\
\hline & 33 & 10 & 721.79 & 1583.88 & 54.43 \\
\hline Overall & & 230 & 17805.2 & 29454.82 & 39.55 \\
\hline
\end{tabular}

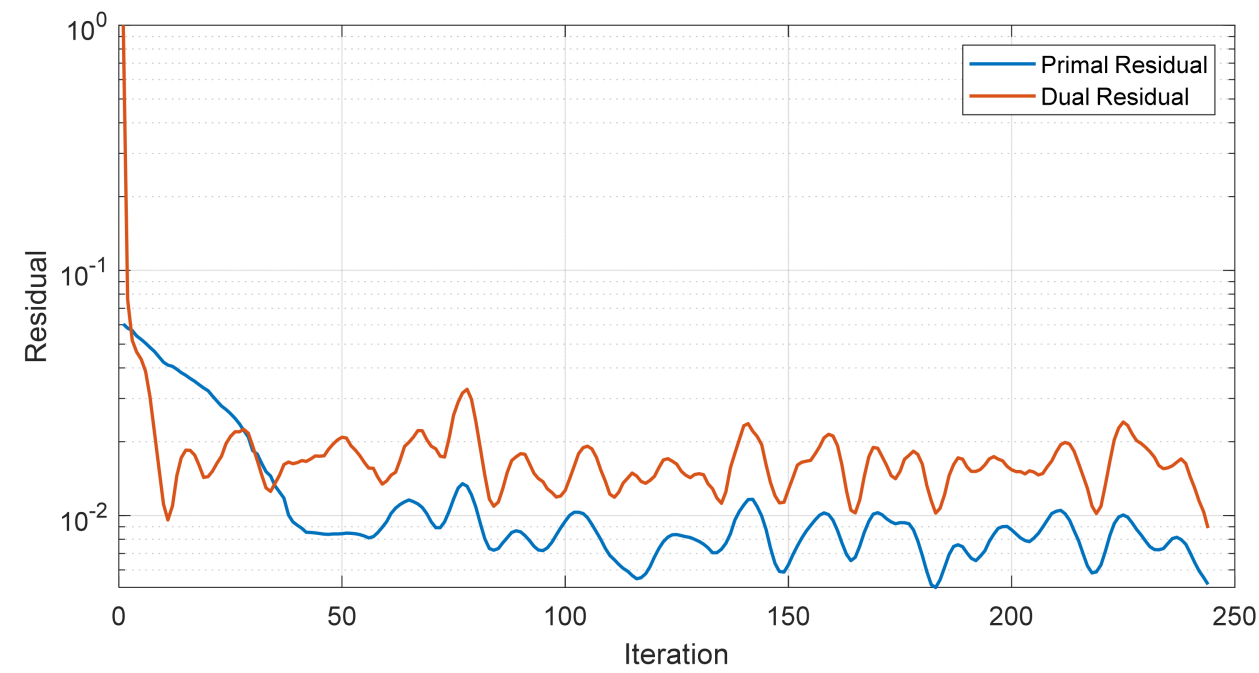

Figure 14. Iteration process of ADMM-based DSO-EVA coordination.

\section{Conclusions}

In this paper, a distributed EV charging schedule with transactive energy management is presented. In the first stage, EVs will propose individual bidding strategies to reflect their charging requirements and cost benefits while the EVAs will generate node-level bidding strategies based on the individual EV bidding information in the node. At the second stage, DSO and EVAs will negotiate to find a balance between the distribution system operation stability and EV charging economic benefits. The DSO ensures network operation with the OPF technique and the EVAs minimize charging costs. In particular, an EV charging price clearing mechanism is developed, which is used in the coordination process. This mechanism provides the incentive for EV charging customers to improve network operation performance. Thus, EVAs and EVs can make their charging scheduling autonomously with clearing price signals. A case study of an IEEE 33-bus distribution feeder demonstrates the effectiveness of the presented TEM algorithms. However, the use case presented in this paper applies certain level-2 AC EV charging. DC fast charging 
and extreme fast charging with battery energy storage systems are not considered. These different charging requirements will cause additional complexity and uncertainty in the distribution grid and will be a focus of our future study. Meanwhile, the fixed building loads are the only consideration in this work. The market-based co-scheduling of the HVAC system and large-scale EV charging control can be further explored for a diversified TE system.

Author Contributions: Conceptualization, Z.W.; methodology, Z.W. and B.C.; software, Z.W.; validation, Z.W; formal analysis, Z.W.; investigation, Z.W.; resources, Z.W.; data curation, Z.W.; writingoriginal draft preparation, Z.W.; writing-review and editing, Z.W. and B.C.; visualization, Z.W.; supervision, B.C.; project administration, B.C.; funding acquisition, B.C. All authors have read and agreed to the published version of the manuscript.

Funding: This research received no external funding.

Institutional Review Board Statement: Not applicable.

Informed Consent Statement: Not applicable.

Data Availability Statement: The data presented in this study are available on request from the corresponding author.

Conflicts of Interest: The authors declare no conflict of interest.

\section{References}

1. Bui, A.; Slowik, P.; Lutsey, N. Power Play: Evaluating the U.S. Position in the Global Electric Vehicle Transition; International Council on Clean Transportation: Washington, DC, USA, 2021. Available online: https://theicct.org/publications/us-position-global-evjun2021 (accessed on 29 July 2021).

2. Jones, C.B.; Lave, M.; Vining, W.; Garcia, B.M. Uncontrolled Electric Vehicle Charging Impacts on Distribution Electric Power Systems with Primarily Residential, Commercial or Industrial Loads. Energies 2021, 14, 1688. [CrossRef]

3. Wang, L.; Cao, C.; Chen, B. Grid-Tied Single-Phase Bi-Directional PEV Charging/Discharging Control. SAE Int. J. Passeng. Cars Electron. Electr. Syst. 2016, 9, 275-285. [CrossRef]

4. Wang, L.; Cao, C.; Chen, B. Model-based micro-grid modeling and optimal PEV charging control. In Proceedings of the 2016 12th IEEE/ASME International Conference on Mechatronic and Embedded Systems and Applications (MESA), Auckland, New Zealand, 29-31 August 2016; pp. 1-6. [CrossRef]

5. Cao, C.; Cheng, M.; Chen, B. Optimal Scheduling of PEV Charging/Discharging in Microgrids with Combined Objectives. Smart Grid Renew. Energy 2016, 7, 115-130. [CrossRef]

6. Cao, C.; Chen, B. Generalized Nash equilibrium problem based electric vehicle charging management in distribution networks. Int. J. Energy Res. 2018, 42, 4584-4596. [CrossRef]

7. Wang, L.; Chen, B. Dual-level consensus-based frequency regulation using vehicle-to-grid service. Electr. Power Syst. Res. 2019, 167, 261-276. [CrossRef]

8. Cao, C.; Wu, Z.; Chen, B. Electric Vehicle-Grid Integration with Voltage Regulation in Radial Distribution Networks. Energies 2020, 13, 1802. [CrossRef]

9. The GridWise Architecture Council. GridWise Transactive Energy Framework Version 1.0; Pacific Northwest National Laboratory: Richland, WA, USA, 2015. Available online: https://www.gridwiseac.org/pdfs/te_framework_report_pnnl-22946 (accessed on 29 July 2021).

10. Chen, S.; Liu, C.C. From demand response to transactive energy: State of the art. J. Mod. Power Syst. Clean Energy 2017, 5, 10-19. [CrossRef]

11. Lezama, F.; Soares, J.; Hernandez-Leal, P.; Kaisers, M.; Pinto, T.; Vale, Z. Local Energy Markets: Paving the Path Toward Fully Transactive Energy Systems. IEEE Trans. Power Syst. 2019, 34, 4081-4088. [CrossRef]

12. Vayá, M.G.; Andersson, G. Optimal Bidding Strategy of a Plug-In Electric Vehicle Aggregator in Day-Ahead Electricity Markets Under Uncertainty. IEEE Trans. Power Syst. 2015, 30, 2375-2385. [CrossRef]

13. Lakshmi, E.S.; Singh, S.P.; Padmanaban, S.; Leonowicz, Z.; Holm-Nielsen, J.B. Prosumer Energy Management For Optimal Utilization of Bid Fulfillment with EV Uncertainty Modeling. IEEE Trans. Ind. Appl. 2021, 1. [CrossRef]

14. Gao, J.; Wong, T.; Wang, C.; Yu, J.Y. A Price-Based Iterative Double Auction for Charger Sharing Markets. IEEE Trans. Intell. Transp. Syst. 2021, 1-12. [CrossRef]

15. Mohammad, A.; Zamora, R.; Lie, T.T. Transactive Energy Management of PV-Based EV Integrated Parking Lots. IEEE Syst. J. 2021, 15, 5674-5682. [CrossRef]

16. Li, J.; Zhang, C.; Xu, Z.; Wang, J.; Zhao, J.; Zhang, Y.J.A. Distributed transactive energy trading framework in distribution networks. IEEE Trans. Power Syst. 2018, 33, 7215-7227. [CrossRef] 
17. Li, Z.; Su, S.; Jin, X.; Chen, H.; Li, Y.; Zhang, R. A hierarchical scheduling method of active distribution network considering flexible loads in office buildings. Int. J. Electr. Power Energy Syst. 2021, 131, 106768. [CrossRef]

18. Hao, H.; Corbin, C.D.; Kalsi, K.; Pratt, R.G. Transactive Control of Commercial Buildings for Demand Response. IEEE Trans. Power Syst. 2017, 32, 774-783. [CrossRef]

19. Wu, Z.; Cao, C.; Chen, B. Transactive Energy Based Approach for Large-Scale Plug-in Electric Vehicle Charging Control. In Proceedings of the 2019 IEEE PES Asia-Pacific Power and Energy Engineering Conference (APPEEC), Macao, China, 1-4 December 2019; pp. 1-5. [CrossRef]

20. Wang, L.; Chen, B. Distributed control for large-scale plug-in electric vehicle charging with a consensus algorithm. Int. J. Electr. Power Energy Syst. 2019, 109, 369-383. [CrossRef]

21. Zhou, Y.; Kumar, R.; Tang, S. Incentive-Based Distributed Scheduling of Electric Vehicle Charging Under Uncertainty. IEEE Trans. Power Syst. 2019, 34, 3-11. [CrossRef]

22. Yan, L.; Chen, X.; Zhou, J.; Chen, Y.; Wen, J. Deep Reinforcement Learning for Continuous Electric Vehicles Charging Control With Dynamic User Behaviors. IEEE Trans. Smart Grid 2021, 12, 5124-5134. [CrossRef]

23. Khaki, B.; Chu, C.; Gadh, R. Hierarchical distributed framework for EV charging scheduling using exchange problem. Appl. Energy 2019, 241, 461-471. [CrossRef]

24. Lin, W.; Jin, X.; Jia, H.; Mu, Y.; Xu, T.; Xu, X.; Yu, X. Decentralized optimal scheduling for integrated community energy system via consensus-based alternating direction method of multipliers. Appl. Energy 2021, 302, 117448. [CrossRef]

25. Farivar, M.; Low, S.H. Branch Flow Model: Relaxations and Convexification-Part I. IEEE Trans. Power Syst. 2013, 28, 2554-2564. [CrossRef]

26. Baran, M.E.; Wu, F.F. Network Reconfiguration in Distribution Systems for Loss Reduction and Load Balancing. IEEE Trans. Power Deliv. 1989, 9, 101-102.

27. Boyd, S.; Parikh, N.; Chu, E.; Peleato, B.; Eckstein, J. Distributed Optimization and Statistical Learning via the Alternating Direction Method of Multipliers. In Foundations and Trends®in Machine Learning; Now Publishers: Delft, The Netherlands, 2011; Volume 3. [CrossRef]

28. EERE. Commercial and Residential Hourly Load Profiles for All TMY3 Locations in the United States, 1st ed. Available online: https:/ / data.openei.org/ (accessed on 20 July 2021).

29. Cao, C.; Wang, L.; Chen, B. Mitigation of the Impact of High Plug-in Electric Vehicle Penetration on Residential Distribution Grid Using Smart Charging Strategies. Energies 2016, 9, 1024. [CrossRef]

30. ComEd. ComEd'S Hourly Pricing Program. Available online: https:/ / hourlypricing.comed.com/ (accessed on 20 July 2021).

31. Lofberg, J. YALMIP: A toolbox for modeling and optimization in MATLAB. In Proceedings of the 2004 IEEE International Conference on Robotics and Automation (IEEE Cat. No.04CH37508), Taipei, Taiwan, 2-4 September 2004; pp. 284-289. [CrossRef] 